\title{
General RM Rings
}

\author{
Chenar Abdul Kareem Ahmed ${ }^{\mathrm{a}, *}$
}

${ }^{a}$ Department of Mathematics, University of Zakho, Kurdistan Region, Iraq, e-mail: chenar . ahmed@uoz. edu .krd

\begin{abstract}
The concept of central RM rings is introduced in this paper as a generalization of RM rings. Since every RM ring is central $\mathrm{RM}$ we study the sufficient condition for a central RM ring to be a RM one. It is shown that every central reversible and hence every central symmetric is central RM ring, however converse implications are wrong. It is also proven that the polynomial ring $R[x]$ is central $R M$ ring if $R$ is central RM and quasi-Armendariz. Also $\alpha$-RM ring has been studied with it is central.@2019 All rights reserved.
\end{abstract}

Keywords: Reflexive ring, Central Reflexive ring, Matrix ring, $\alpha$-Rigid ring, Dorroh extension. 2010 MSC: 16S70, 16S80, 16U80

\section{Introduction}

Throughout this paper all rings are associative with unity unless otherwise sated. For a ring $R$, $M_{n}(R), T_{n}(R), Z(R), R[x], \alpha$ and $i d_{R}$ denote the $n \times n$-full matrix ring over $R, n \times n$-upper triangular matrix ring over $R$, the center of $R$, the polynomial ring over $R$, an endomorphism and the identity endomorphism of $R$ respectively. Denote $\left\{\left(a_{i j}\right) \in U_{n}(R) \mid\right.$ the diagonal entries of $\left(a_{i j}\right)$ are all equal $\}$ by $D_{n}(R)$. By $\mathbb{Z}$, we mean the ring of integers unless otherwise mentioned. A ring is called reduced if it has no nozero nilpotent element, similarly a ring $R$ is called central reduced [16] if every nilpotent element of $\mathrm{R}$ is central.

A ring $R$ is said to be symmetric [31] if for any $a, b, c \in R$ with $a b c=0$ implies $a c b=0$ if and only if $a b c=0$ implies $b a c=0$. it can be equivalent defined as whenever a product of any number of elements of $R$ is zero, then any permutation of the factors gives product zero [5]. Kafkas et al. [21] defined that a ring $R$ is called central symmetric if whenever $a b c=0$ for $a, b, c \in R$ then $b a c \in Z(R)$. They have shown that the class of symmetric rings is strictly contained in the class of centeral symmetric rings. A ring $R$ is said to be reversible [12] if $a b=0$ for $a, b \in R$, then $b a=0$, similarly a ring $R$ is called central reversible [21] if for any $a, b \in R, a b=0$ implies $b a \in Z(R)$. It is shown that every symmetric is reversible and every central symmetric ring is central reversible, however converses in both occasions are not true. A ring $R$ is called semicommucative if whenever $a b=0$ for $a, b \in R$, then $a R b=0$, similarly a ring $R$ is defined to be central semicommutative [34] if $a b=0$ for $a, b \in R, a R b=(0)$ implies $b R a=(0)$. Both the classes of semicommuative rings and reflexive rings contain the class of reversible rings and the inclusions

\footnotetext{
*Corresponding author

Email address: Chenar Abdul Kareem Ahmed@example.com (Chenar Abdul Kareem Ahmed)
} 
are strict, in general. Moreover, semicommutaive rings and reflexive rings donot imply each other [26, Example 2.3 and Theorem 2.6(2)]. Motivated by these, we introduce the concept of centreal RM rings as a generalization of RM rings and central reversible rings. The relation among all the central versions of rings mentioned above can be given as follows:

On the other hand, an endomorphism $\alpha$ of a ring $R$ is called rigid [3] if $a \alpha(a)=0$ implies $a=0$ for $a \in R$ and $R$ is called an $\alpha$-rigid ring [16] if there exists such a rigid endomorphism $\alpha$ of $R$. Baser et al. defined $\alpha$-reversible [6] and $\alpha$-semicommuative rings [7] as a generalization of $\alpha$-rigid rings. Zhao and $\mathrm{Zhu}$ [37] defined a ring to be $\alpha$-reflexive if there exists an endomorphism $\alpha: R \rightarrow R$ such that for $a, \in R$ if $a R b=(0)$, then $b R \alpha(a)=0$.

A ring $R$ is called semi prime if $a R a=0$ for every $a \in R$ implies $a=0$, every semiprime rings is reduced but clearly the converse is not true.

Generalized reduced rings were extended by ring endomorphisms. According to Krempa [16], an endomorphism $\alpha$ of a ring $R$ is called rigid if $a \alpha(a)=0$ implies $a=0$ for $a \in R$, and a ring $R$ is called $\alpha$-rigid [16] if there exists a rigid endomorphism $\alpha$ of $R$. Note that any rigid endomorphism of a ring is a monomorphism and $\alpha$-rigid rings are reduced rings by [16, Proposition 5]. By Hashemi and Moussavi [14], a ring $R$ is $\alpha$-compatible if for each $a, b \in R, a \alpha(b)=0$ if and only if $a b=0$, every $\alpha$-rigid ring is $\alpha$-compatible but the converse is not true.

In [6, Definition 2.1], an endomorphism $\alpha$ of a ring $R$ is called right skew reversible if whenever $a b=0$ for $a, b \in R, b \alpha(a)=0$, and the ring $R$ is called right $\alpha$-skew reversible if there exists a right skew reversible endomorphism $\alpha$ of R. Similarly, left $\alpha$-skew reversible rings are defined. A ring $R$ is called $\alpha$-skew reversible if it is both left and right $\alpha$-skew reversible. Note that $R$ is an $\alpha$-rigid ring if and only if $R$ is semiprime and right $\alpha$-skew reversible for a monomorphism $\alpha$ of $R$ by [6, Proposition 2.5(iii)]. (We change over from "an $\alpha$-reversible ring" in [6] to "an $\alpha$-skew reversible ring" to cohere with other related definitions.)

The reflexive property was studied by Mason [22]. Mason [22] called a right ideal I of a ring R (possibly without identity) reflexive if $a R b \subseteq$ I implies $b R a \subseteq I$ for $a, b \in R$, and a ring $R$ is usually called reflexive if 0 is a reflexive ideal (i.e., $a R b=0$ implies $b R a=0$ for $a, b \in R$ ).

In [28, Definition 2.1], an endomorphism $\alpha$ of a ring $R$ is called right (resp., left) skew reflexive if whenever $a R b=0$ for $a, b \in R, b R \alpha(a)=0$, and the ring $R$ is called right (resp., left) $\alpha$-skew reflexive if there exists a right (resp., left) skew reflexive endomorphism $\alpha$ of $R$. A ring $R$ is $\alpha$-skew reflexive if it is both right and left $\alpha$-skew reflexive. A ring $R$ is reflexive if $R$ is $1_{R}$-reflexive ring where $1_{R}$ denotes the identity endomorphism of $R$. Any domain $R$ is obviously $\alpha$-skew reflexive for any endomorphism $\alpha$ of $R$, but the converse need not hold by the [28, Example 2.2]. An endomorphism $\alpha$ of a ring $\mathrm{R}$ is called right (resp., left) skew RIP if whenever $e \mathrm{Rf}=0$ for $e, f \in \operatorname{Id}(\mathrm{R}), f R \alpha(e)=0$, and the ring $\mathrm{R}$ is called right (resp., left) $\alpha$-skew $R I P$ if there exists a right (resp., left) skew RIP endomorphism $\alpha$ of R. A ring $R$ is $\alpha$-skew RIP if it is both right and left $\alpha$-skew RIP.

A ring $R$ is said to have the reflexivity with maximal ideal [1] if $R$ satisfies the property that

$$
\mathrm{aMb}=0 \text { whenever } \mathrm{bMa}=0 \text { for a maximal ideal } M \text { of } \mathrm{R} \text { and } \mathrm{a}, \mathrm{b} \in \mathrm{R} . \quad(*)
$$

A ring is called $R M$ if it satisfies the condition $(*)$.

We will freely use these facts without reference.

Motivated by the above facts, the concepts of Central RM and Central $\alpha-\mathrm{RM}$ rings have been introduced, as a generalization of $\alpha$-rigid rings. First basic examples and properties has been found. Also Central RM property of some kind of polynomials have been discussed.

Throughout this paper, $\alpha$ denotes a nonzero endomorphism of given rings, unless specified otherwise.

Motivated by the above facts, the concepts of Central RM and Central $\alpha$-RM rings have been introduced, as a generalization of $\alpha$-rigid rings. First basic examples and properties has been found. Also 
Central RM property of some kind of polynomials have been discussed.

2. Central RM rings

In this section properties of central RM rings and related examples are found .

Definition 2.1. A ring $R$ is said to be central $R M$ for any $a, b \in R$ and for a maximal ideal $M$ of $R$, whenever $a M b=(0)$, then $b M a \subset Z(R)$.

All RM rings are clearly central RM. But the converse is not true by the following example.

Let $K$ be a field and $A=K\langle x, y, z\rangle$ be the free algebra with non-commuting three indeterminates $x, y, z$ over K. Let I be the ideal of A generated by $x^{2}, y^{2}, z^{2}$, all quadratic products of $x, y, z$ except $x y, y x z, z y x$, and all quartic products of $x, y, z$. Set $R=A / I=\left\{a_{0}+a_{1} x+a_{2} y+a_{3} z: a_{0}, a_{1}, a_{2}, a_{3} \in \mathbb{K}\right\}$. Identify the elements in $A$ with their images in $R$ for simplicity. The ring $R$ is central RM by straightforward competion. But $R$ is not $R M$ by the following computation. Let $M$ be the ideal of $R$ generated by $x, y$ and $z$. Then $M$ is the unique maximal ideal of $R$ since $R / M \cong K$. We have $y M x=0$, but $0 \neq x z y \in x M y$. Thus $\mathrm{R}$ is not $\mathrm{RM}$.

Now we find some condition under which a central RM ring is RM. For a nonempty subset $S$ of a ring $R, r_{R}(S)=\{c \in R \mid S c=0\}$ is called the right annihilator of $S$ in $R$. The left annihilator is defined similarly and written by $\ell_{R}(S)$. If $S=\{a\}$, then we we write $r_{R}(a)$ (resp., $\ell_{R}(a)$ ) instead of $r_{R}(\{a\})$ (resp., $\ell_{R}(\{a\})$ ). Recall that a ring $R$ is called right(left) principally quasi-Baer [9] if the right (left) annihilator of a principal right (left) ideal of $R$ generated by an idempotent. A ring $R$ is called right (left) principally projective [10] if the right (left) annihilator of an element of $R$ is generated by an idempotent.

Proposition 2.2. Let $\mathrm{R}$ be a central $R M$ ring. Then $\mathrm{R}$ is $\mathrm{RM}$ if any of the following conditions holds:

(i) $R$ is a right(left) principally projective ring.

(ii) $\mathrm{R}$ is a right(left) principally quasi-Baer ring.

Proof. Suppose that $a M b=(0)$ for $a, b \in R$ and for a maximal idea $M$. Then bma $\in Z(R)$ for any $m \in M$, since $R$ is central RM.

(i) Let $R$ be a right principally projective ring. Then there is an idempotent $e \in R$ such that $r_{R}(a)=e M$. Thus $a e=0$. Since $b m \in r_{R}(a)=e M$, we have $b m=e b m$. It follows that $b m a=e b m a=b m a e=0$, since $b m a \in Z(R)$ and $a e=0$. Therefore $R$ is $R M$. A similar proof may be given for left principally projective rings.

(ii) Let $\mathrm{R}$ be a right(left) principally quasi-Baer ring. Then there is an idempotent $e \in R$ such that $b m \in r_{R}(a)=e M$. Thus $a e=0$. On the other hand, $a x b m a=a b m a x=0$ for all $x \in R$, since $a M b=(0)$ and bma $\in Z(R)$. This implies that bma $\in r_{R}(a M)=(e M)$, and so bma $=e b m a=b m a e=0$, by the same method as the proof of (i). Thus $R$ is RM. Similarly it can be shown that every left principally quai-Baer central RM ring is RM.

Proposition 2.3. Let $\mathrm{R}$ be a ring. Then the following statements are equivalent:

(1) $R$ is a central RM ring.

(2) For a maximal ideal $M$ of $R, A M B=0$ implies $B M A \subset Z(R)$ where $A$ and $B$ are any nonempty subsets of R.

(3) For a maximal ideal $M$ of $R, I M J=0$ implies $J M I \subset Z(R)$ where $I$ and $J$ are any right (or, left) ideals of $R$.

(4) For a maximal ideal $M$ of $R, I M J=0$ implies $J M I \subset Z(R)$ where $I$ and $J$ are any ideal of $R$.

Proof. (1) $\Rightarrow(2)$ Assume that the condition (i) holds. Let $A$ and $B$ be two nonempty subsets of $R$ with $A M B=0$ for a maximal ideal $M$ of $R$. Then $a M b=0$ for any $a \in A$ and $b \in B$, and hence $b M a \subset Z(R)$ by assumption. Thus, $B M A=\sum_{a \in A, b \in B} b M a \subset Z(R)$. 
$(2) \Rightarrow(3)$ Let $I$, J be two right ideals of R such that $I M J=(0)$, for a maximal ideal $M$ of $R$. By (2) we have $J M I \subset Z(R)$. A similar proof may be given for left ideals of $R$.

(3) $\Rightarrow(4)$ is straightforward.

$(4) \Rightarrow(1)$ Assume that the condition (4) holds. Let $a M b=0$ for a maximal ideal $M$ of $R$ and $a, b \in R$. Then $a R M R b \subseteq a M b=0$ and so RaRMRbR $=0$. By assumption, we have $R b R M R a R \subset Z(R)$ and hence $b M a \subset Z(R)$. Thus $R$ is a central RM ring.

We know that every reduced ring is RM. The same follows for their central version also.

Proposition 2.4. If $\mathrm{R}$ is reduced ring, then it is central $R M$.

Proof. Let $R$ be reduced ring and let $a, b \in R$ and for a maximal ideal $M$ of $R$ such that $a M b=(0)$. Then $a m b=b a m=0$ for $m \in M$ by assumption. Then bma $\subset Z(R)$. Hence $b M a \subset Z(R)$. Thus $R$ is central RM.

The converse of the above result is however not true as shown by the following example.

The ring $M_{2}(Z)$ is Reflexive [26, Example 1.6] and hence central RM. But $M_{2}(Z)$ is not reduced for $0 \neq A=\left(\begin{array}{ll}0 & 1 \\ 0 & 0\end{array}\right) \in M_{2}(Z)$ but $A^{2}=0$.

Subring of central RM ring may not be central RM, which can be shown by the following example.

Consider the central RM ring as in Example 2. But $T_{n}(Z)$ is subring of $M_{n}(Z)$, which is not central $\mathrm{RM}$ as

$$
\left(\begin{array}{ll}
0 & 1 \\
0 & 1
\end{array}\right)\left(\begin{array}{ll}
a & b \\
0 & c
\end{array}\right)\left(\begin{array}{ll}
1 & 1 \\
0 & 0
\end{array}\right)=0
$$

for all $\left(\begin{array}{ll}a & b \\ 0 & c\end{array}\right) \in M\left(T_{n}\right)(Z)$, but

$$
\left(\begin{array}{ll}
1 & 1 \\
0 & 0
\end{array}\right)\left(\begin{array}{ll}
1 & 2 \\
0 & 3
\end{array}\right)\left(\begin{array}{ll}
0 & 1 \\
0 & 1
\end{array}\right)=\left(\begin{array}{ll}
0 & 6 \\
0 & 0
\end{array}\right) \notin Z\left(T_{n}(Z)\right) .
$$

The ring $M_{2}(Z)$ is $R M\left[26\right.$, Example 1.6] and hence central RM. But $M_{2}(Z)$ is not reduced for $0 \neq A=$ $\left(\begin{array}{ll}0 & 1 \\ 0 & 0\end{array}\right) \in M_{2}(Z)$ but $A^{2}=0$.

One may conjecture that $M_{n}(Z)$ is central $R M$ if $R$ is central RM. But the following example erases that possibility.

Consider the ring $R$ in Example 2 in the present study. here $R$ is central $R M$, but $M_{n}(R)$ is not so, as

$$
\begin{gathered}
\qquad\left(\begin{array}{ll}
y & y \\
0 & 0
\end{array}\right)\left(\begin{array}{cc}
a_{0}+a_{1} x+a_{2} y+a_{3} z & b_{0}+b_{1} x+b_{2} y+b_{3} z \\
c_{0}+c_{1} x+c_{2} y+c_{3} z & c_{0}+c_{1} x+c_{2} y+c_{3} z
\end{array}\right)\left(\begin{array}{ll}
x & 0 \\
0 & 0
\end{array}\right)=0, \\
\text { for all }\left(\begin{array}{cc}
a_{0}+a_{1} x+a_{2} y+a_{3} z & b_{0}+b_{1} x+b_{2} y+b_{3} z \\
c_{0}+c_{1} x+c_{2} y+c_{3} z & c_{0}+c_{1} x+c_{2} y+c_{3} z
\end{array}\right) \in \mathcal{N}\left(M_{n}(R)\right) \text { but } \\
\left(\begin{array}{cc}
x & 0 \\
0 & 0
\end{array}\right)\left(\begin{array}{ll}
z & z \\
0 & 0
\end{array}\right)\left(\begin{array}{ll}
y & y \\
0 & 0
\end{array}\right) \notin Z\left(M_{n}(Z)\right) .
\end{gathered}
$$

Proposition 2.5. (1) If $\mathrm{R}$ is a central $R M$ ring then so is eRe for all $e \in \operatorname{Id}(\mathrm{R})$.

(2) If $M_{n}(R)$ over any ring $R$ is central $R M$ for $n \geqslant 2$, then so is $R$. RM.

(3) Let $R$ be a ring and $e \in \operatorname{Id}(R)$. If $e$ is central then $R$ is central $R M$ if and only if e $R$ and $(1-e) R$ are central 
Proof. (1) Let $e \in \operatorname{Id}(R)$. First, we claim that $M$ is a maximal ideal of $e$ Re if and only if $M=e N e$ for some maximal ideal $N$ of $R$. Let $M$ be a maximal ideal of $e$ Re. Then clearly $M=e N e$ for some ideal $N$ of $R$. We show that $\mathrm{N}$ is a maximal ideal of $\mathrm{R}$. Assume on the contrary that there exists an ideal $\mathrm{S}$ of $\mathrm{R}$ such that $\mathrm{N} \subsetneq \mathrm{S} \subsetneq \mathrm{R}$. Take $r_{0} \in \mathrm{R}$ but $r_{0} \notin \mathrm{S}$. Then $M=e \mathrm{Ne} \subsetneq e S e$ and $e S e$ is an ideal of $e \mathrm{Re}$, and so $e S e=e \mathrm{Re}$ since $M$ is a maximal ideal of eRe. But it is a contradiction to $e r_{0} e \notin e S e$ for $r_{0} \in R$. Thus $M=e N e$ and $\mathrm{N}$ is a maximal ideal of $\mathrm{R}$.

One can see the proof of the converse of claim in the literature. Now, let $R$ be a central $R M$ ring and $e \in \operatorname{Id}(R)$. Suppose $a M b=0$ for $a, b \in e R e$ and a maximal ideal $M$ of $e$ Re. Note that $a e=a=e a$ and $e b=b=b e$. By claim, there exists a maximal ideal $N$ of $R$ such that $M=e N e$. Hence, $0=a M b=$ $a e N e b=a N b$, and so $b N a \subset Z(R)$ since $R$ is central RM. Thus $Z(R) \supset b N a=b e N e a=b M a$, showing that $e$ Re is central RM.

(2) It comes from (1), since $R \cong R e_{11}=e_{11} M_{n}(R) e_{11}$ where $e_{11} \in \operatorname{Id}\left(M_{n}(R)\right)$.

(3) Let $e \in \operatorname{Id}(R)$ be central. Suppose that $e R$ and $(1-e) R$ are central $R M$ and let $a M b=0$ for $a, b \in R$ and a maximal ideal $M$ of $R$. Recall that $e M$ and $(1-e) M$ are maximal ideals of $e R$ and $(1-e) R$ respectively, by the proof of $(1)$. Then $a(e M) b=e a M b=0$ and $a((1-e) M) b=(1-e) a M b=0$. By hypothesis, we have $b(e M) a=e b M a \subset Z(R)$ and $b((1-e) M) a=(1-e) b M a \subset Z(R)$. Thus $b M a=$ $e b M a+(1-e) b M a \subset Z(R)$, showing that $R$ is central RM.

The converse follows from (1) directly.

The converse of Proposition 2.5(1) does not hold in general. The non-RM ring $R=T_{2}(\mathbb{Z})$. For $e=e_{11} \in \operatorname{Id}(R), e R e \cong \mathbb{Z}$ is $R M$ clearly.

Recall that for a ring $R$ and an $(R, R)$-bimodule $M$, the trivial extension of $R$ by $M$ is the ring $T(R, M)=$ $R \oplus M$ with the usual addition and the following multiplication: $\left(r_{1}, m_{1}\right)\left(r_{2}, m_{2}\right)=\left(r_{1} r_{2}, r_{1} m_{2}+m_{1} r_{2}\right)$. This is isomorphic to the ring of all matrices $\left(\begin{array}{cc}r & m \\ 0 & r\end{array}\right)$, where $r \in R$ and $m \in M$ and the usual matrix operations are used. Note that $T(R, R)=D_{2}(R)$.

Recall that for a ring $R$ and an $(R, R)$-bimodule $M$, the trivial extension of $R$ by $M$ is the ring $T(R, M)=$ $R \oplus M$ with the usual addition and the following multiplication: $\left(r_{1}, m_{1}\right)\left(r_{2}, m_{2}\right)=\left(r_{1} r_{2}, r_{1} m_{2}+m_{1} r_{2}\right)$. This is isomorphic to the ring of all matrices $\left(\begin{array}{cc}r & m \\ 0 & r\end{array}\right)$, where $r \in R$ and $m \in M$ and the usual matrix operations are used. Note that $T(R, R)=D_{2}(R)$.

Recall that $T(R, R)$ of a semiprime ring $R$ is ideal-symmetric by help of [11, Corollary 2.9] and thus RM. Since every RM ring is central RM, therefore we can say that if $R$ is semiprime, then $T(R, R)$ is central RM. Moreover we obtain the following.

Theorem 2.6. (1) If $\mathrm{R}$ is a simple ring, then $\mathrm{T}(\mathrm{R}, \mathrm{R})$ is central $R M$.

(2) If $T(R, R)$ of any ring $R$ is central $R M$, then so is $R$.

(3) If $M_{n}(R)$ over any ring $R$ is central $R M$ for $n \geqslant 2$, then so is $R$.

Proof. (1) Let R be simple. Then

$$
M=\left(\begin{array}{cc}
0 & R \\
0 & 0
\end{array}\right)
$$

is the only maximal ideal of $T(R, R)$. Suppose that $A M B=0$ for

$$
A=\left(\begin{array}{ll}
a & b \\
0 & a
\end{array}\right), B=\left(\begin{array}{ll}
c & d \\
0 & c
\end{array}\right) \in T(R, R) .
$$

Then we have $a R c=0$. But since $R$ is simple, $R$ is $R M$ and so $R$ is central $R M$ and we get $c R a \subset Z(R)$. This implies that $B M A \subset Z(R)$. Thus $T(R, R)$ is central RM. 
(2) Suppose that $T(R, R)$ of any nonzero ring $R$ is central $R M$ and let $a M b=0$ for $a, b \in R$ and $a$ maximal ideal $M$ of $R$. Then

$$
\left(\begin{array}{cc}
M & R \\
0 & M
\end{array}\right)
$$

is a maximal ideal of $T(R, R)$ and

$$
\left(\begin{array}{ll}
\mathrm{a} & 0 \\
0 & \mathrm{a}
\end{array}\right)\left(\begin{array}{cc}
\mathrm{M} & \mathrm{R} \\
0 & \mathrm{M}
\end{array}\right)\left(\begin{array}{ll}
0 & \mathrm{~b} \\
0 & 0
\end{array}\right)=\left(\begin{array}{cc}
0 & \mathrm{aMb} \\
0 & 0
\end{array}\right)=0
$$

Since $T(R, R)$ is $R M$,

$$
\left(\begin{array}{ll}
0 & b \\
0 & 0
\end{array}\right)\left(\begin{array}{cc}
M & R \\
0 & M
\end{array}\right)\left(\begin{array}{ll}
a & 0 \\
0 & a
\end{array}\right)=\left(\begin{array}{cc}
0 & b M a \\
0 & 0
\end{array}\right) \subset \mathrm{Z}(T(R, R))
$$

and so $b M a \subset Z(R)$. Thus $R$ is central $R M$.

Theorem 2.7. If $\mathrm{T}(\mathrm{R}, \mathrm{R})$ is a central $\mathrm{RM}$ ring, then $\mathrm{R}$ is central reflexive ring.

Proof. Let $T(R, R)$ be central RM. First, suppose that $a R b=0$ for $a, b \in R$. Then $a M b=0$ for a maximal ideal $M$ of $R$. Since $\mathcal{M}=\left(\begin{array}{cc}M & R \\ 0 & M\end{array}\right)$ is a maximal ideal of $T(R, R)$ and $\left(\begin{array}{ll}a & 0 \\ 0 & a\end{array}\right) \mathcal{M}\left(\begin{array}{ll}b & 0 \\ 0 & b\end{array}\right)=0$, we have $\left(\begin{array}{ll}b & 0 \\ 0 & b\end{array}\right) \mathcal{M}\left(\begin{array}{ll}a & 0 \\ 0 & a\end{array}\right) \subset Z(T(R, R))$, by hypothesis. This yields $b R a \subset Z(R)$, and so $R$ is central reflexive.

The following example shows that there exists a ring $R$ which is not central RM such that for any nonzero proper ideal $I$ of $R, R / I$ is central $R M$ and $I$ is central $R M$ as a ring without identity.

Consider $R=D_{3}(F)$ over a field $F$. Then $R$ is not RM by [1, Example 1.7(2)]. All the nonzero proper ideals of $R$ are $I_{1}=\left(\begin{array}{lll}0 & F & F \\ 0 & 0 & F \\ 0 & 0 & 0\end{array}\right), I_{2}=\left(\begin{array}{lll}0 & F & F \\ 0 & 0 & 0 \\ 0 & 0 & 0\end{array}\right), I_{3}=\left(\begin{array}{lll}0 & 0 & F \\ 0 & 0 & F \\ 0 & 0 & 0\end{array}\right), I_{4}=\left(\begin{array}{ccc}0 & 0 & F \\ 0 & 0 & 0 \\ 0 & 0 & 0\end{array}\right)$ and $I_{5}=$ $\left\{\left(\begin{array}{ccc}0 & \alpha b & c \\ 0 & 0 & \alpha d \\ 0 & 0 & 0\end{array}\right) \mid b, c, d, \alpha \in F\right.$ and $b \neq 0, d \neq 0$ are fixed $\}$. Each $I_{i}$ for $i=1,2,3,4,5$ is nilpotent of index $\leqslant 3$, and so it is clearly $R M$, but they are not reduced obviously. Note that $R / I_{1} \cong F$ is $R M$ and $R / I_{5} \cong$ $D_{2}(F)$ is also RM by help of Theorem 3.10(2). Now we show that every $R / I_{j}$ for $j=2,3,4$ is $R M$ based on the condition that $F$ is a field. Let $\bar{R}_{j}=R / I_{j}$ for $j=2,3,4$. Then $\bar{R}_{2}=\left\{\left(\begin{array}{lll}a & 0 & 0 \\ 0 & a & d \\ 0 & 0 & a\end{array}\right)+I_{2} \mid a, d \in F\right\}, \bar{R}_{3}=$ $\left\{\left(\begin{array}{lll}a & b & 0 \\ 0 & a & 0 \\ 0 & 0 & a\end{array}\right)+I_{3} \mid a, b \in F\right\}$ and $\bar{R}_{4}=\left\{\left(\begin{array}{lll}a & b & 0 \\ 0 & a & d \\ 0 & 0 & a\end{array}\right)+I_{4} \mid a, b, d \in F\right\}$. Each $\bar{M}_{j}$ in the following is only a maximal ideal of $\bar{R}_{j}$ for $j=2,3,4$ respectively, since $F$ is a field. $\bar{M}_{2}=\left\{\left(\begin{array}{lll}0 & 0 & 0 \\ 0 & 0 & d \\ 0 & 0 & 0\end{array}\right)+I_{2} \mid \mathrm{d} \in \mathrm{F}\right\}, \bar{M}_{3}=$ $\left\{\left(\begin{array}{lll}0 & \mathrm{~b} & 0 \\ 0 & 0 & 0 \\ 0 & 0 & 0\end{array}\right)+\mathrm{I}_{3} \mid \mathrm{b} \in \mathrm{F}\right\}$ and $\bar{M}_{4}=\left\{\left(\begin{array}{lll}0 & 0 & 0 \\ 0 & 0 & 0 \\ 0 & 0 & 0\end{array}\right)\right\}$. Then it can be easily checked that each $\overline{\mathrm{R}}_{\mathrm{j}}=$ $R / I_{j}$ for $j=2,3,4$ is $R M$.

Therefore every $R / I_{i}$ (for $i=1,2,3,4,5$ ) is $R M$.

Proposition 2.8. Let $\mathrm{R} / \mathrm{I}$ be a central RM ring for any ideal $\mathrm{I}$ of a ring $\mathrm{R}$. If $\mathrm{I}$ is a reduced as a ring without identity, then $\mathrm{R}$ is central RM. 
Proof. Let $a M b=0$ with $a, b \in R$ and for a maximal ideal $M$ of $R$. Since $R / I$ is central $R M$ and $M / I$ is a maximal ideal of $R / I$. Hence $a M b+I=I \rightarrow b M a+I \subset Z(R / I)$. That is $b M a R-R b M a \subseteq I$. Now let bmar $-r b m a \in I$ for all $m \in M$ and $r \in R$. So $(b m a r-r b m a)^{2} \in($ bmar $-r b m a) I=0$. Since $I$ is reduced we get $b$ mar $-r b m a=0$. Therefore $b m a r=r b m a$ for all $m \in M$ and $r \in R$ and $b M a R=R b M a$ implies $b M a \subset Z(R)$. Hence $R$ is central $R M$ ring.

An element $u$ of a ring $R$ is right regular if $u r=0$ implies $r=0$ for $r \in R$. Similarly, left regular elements can be defined. An element is regular if it is both left and right regular (i.e., not a zero divisor).

A multiplicatively closed subset $S$ of a ring $R$ is said to satisfy the right Ore condition if for each $a \in R$ and $b \in S$, there exist $a_{1} \in R$ and $b_{1} \in S$ such that $a b_{1}=b a_{1}$. It is shown by [33, Theorem 2.1.12] that $S$ satisfies the right Ore condition and $S$ consists of regular elements if and only if the right quotient ring $\mathrm{Q}(\mathrm{R})$ of $\mathrm{R}$ with respect to $\mathrm{S}$ exists.

Theorem 2.9. Let $\Delta$ be a multiplicatively closed subset of a ring $\mathrm{R}$ consisting of central regular elements. Then $\mathrm{R}$ is central $R M$ if and only if $\Delta^{-1} \mathrm{R}$ is central $R M$.

Proof. Let $\mathrm{R}$ be a central RM ring and let $a u^{-1}, b v^{-1} \in \Delta^{-1} \mathrm{R}$ such that $a u^{-1} \mathcal{M}\left(\Delta^{-1} \mathrm{R}\right) b v^{-1}=(0)$. Thus for any $\mathrm{ms}^{-1} \in M\left(\Delta^{-1} \mathrm{R}\right)=\Delta^{-1}(\mathcal{M}(\mathrm{R}))$, we have $a u^{-1} \mathrm{~ms}^{-1} b v^{-1}=0$. This implies $a \mathrm{mb}=0$ and so $a M b=0$ for a maximal idead $M$ of $R$, and so $a M b=(0)$. Since $R$ is central $R M$, therefore $b M a \subset Z(R)$, i.e.bma $\in Z(R)$. Since $u^{-1}, v^{-1}$ and $s^{-1}$ are central elements, therefore $b v^{-1} m s^{-1} a u^{-1} \in Z\left(\Delta^{-1} R\right)$. Thus $b v^{-1} \Delta^{-1}(\mathcal{M} R) a u^{-1} \subset Z\left(\Delta^{-1} R\right)$, and hence $\Delta^{-1} R$ is central RM.

Conversely, assume that $\Delta^{-1} R$ is central RM. For $a, b \in R$ and for maximal ideal $M$ of $R$ let $a M b=0$. Thus $a \Delta^{-1}(\mathcal{M} R) b=0$. Since $\Delta^{-1} R$ is central $R M$, therefore $b \Delta^{-1}(\mathcal{M} R) a \subset Z(R)$. Therefore $b M a \subset Z(R)$. Hence $R$ is central RM.

Proposition 2.10. Let $\mathrm{R}$ be a ring. Then $\mathrm{R}[\mathrm{x}]$ is central $R M$ if and only if $\mathrm{R}\left[\mathrm{x}, \mathrm{x}^{-1}\right]$ is central $R M$.

Proof. Let $\Delta=1, x, \chi^{2}, \ldots$. Then clearly $\Delta$ is a multiplicative closed subset of $R[x]$ and $R\left[x, x^{-1}\right]=\Delta^{-1} R[x]$. The rest follows from Theorem 2.15

A ring $R$ is called (von Neumann) regular if for each $a \in R$ there exists $b \in R$ such that $a=a b a$. It is well-known that a regular ring $R$ is reversible if and only if it is IFP if and only if it is Abelian.

Let $R$ be an algebra over a commutative ring $S$. Due to Dorroh [13], the Dorroh extension of $R$ by $S$ is the Abelian group $R \oplus S$ with multiplication given by $\left(r_{1}, s_{1}\right)\left(r_{2}, s_{2}\right)=\left(r_{1} r_{2}+s_{1} r_{2}+s_{2} r_{1}, s_{1} s_{2}\right)$ for $r_{i} \in R$ and $s_{i} \in S$. We use $D$ to denote the Dorroh extension of $R$ by $S$.

For an algebra $R$ over a commutative ring $S$, the Dorroh extension $D$ of $R$ by $S$ is the Abelian group $D=R \oplus S$ with multipication given by $\left(r_{1}, s_{1}\right)\left(r_{2}, s_{2}\right)=\left(r_{1} r_{2}+s_{1} r_{2}+s_{1} r_{2}+s_{2} r_{1}, s_{1} s_{2}\right)$, where $r_{i} \in R$ and $s_{i} \in S$.

Theorem 2.11. Let $\mathrm{R}$ be an algebra over a commutative $\mathrm{S}$. Then $\mathrm{R}$ is central $\mathrm{RM}$ if and only if the Dorroh extension of $\mathrm{R}$ by $\mathrm{S}$ is so.

Proof. First, note that $\mathcal{M}=R \oplus\{0\}$ is the unique maximal ideal of $D$, since every $(r, s) \in D$ is a unit when $s \neq 0$ by the proof of [19, Proposition 1.5] and that $s \in S$ is identified with $s 1 \in R$ and so $R=\{r+s \mid$ $(\mathrm{r}, \mathrm{s}) \in \mathrm{D}\}$.

Suppose that $R$ be central RM and let $\left(r_{1}, s_{1}\right) \mathcal{M}\left(r_{2}, s_{2}\right)=0$ for $\left(r_{1}, s_{1}\right),\left(r_{2}, s_{2}\right) \in D$. Since

$$
\left(r_{1}, s_{1}\right)(r, 0)\left(r_{2}, s_{2}\right)=\left(r_{1} r r_{2}+s_{1} r r_{2}+s_{2} r_{1} r+s_{1} s_{2} r, 0\right)=(0,0),
$$

for $r_{1}, r, r_{2} \in R, s_{1}, s_{2} \in S$ and 


$$
\left(r_{1}+s_{1} 1\right) r\left(r_{2}+s_{2} 1\right)=r_{1} r r_{2}+s_{1} r r_{2}+s_{2} r_{1} r+s_{1} s_{2} r
$$

for $\left(r_{1}+s_{1} 1\right),\left(r_{2}+s_{2} 1\right) \in R$ and $r \in R$.

For any $(r, 0) \in \mathcal{M}$, we have $\left(r_{1}, s_{1}\right) R\left(r_{2}, s_{2}\right)=0$. Since $R$ is a right $\alpha$-skew reflexive ring, we get

$$
0=\left(r_{2}+s_{2} 1\right) R \alpha\left(r_{1}+s_{1} 1\right)=\left(r_{2}+s_{2} 1\right) R\left(\alpha\left(r_{1}\right)+s_{1}\right) .
$$

Hence

$$
r_{2} r \alpha\left(r_{1}\right)+s_{2} r \alpha\left(r_{1}\right)+s_{1} r_{2} r+s_{1} s_{2} r=0,
$$

for all $r \in R$. Let $(r, 0) \in \mathcal{M}$. Then

$\left(r_{2}, s_{2}\right)(r, 0)\left(\alpha\left(r_{1}\right), s_{1}\right)=\left(\left(r_{2} r+s_{2} r\right) \alpha\left(r_{1}\right)+s_{1}\left(r_{2} r+s_{2} r\right), 0\right)=\left(r_{2} r \alpha\left(r_{1}\right)+s_{2} r \alpha\left(r_{1}\right)+s_{1} r_{2} r+s_{1} s_{2} r, 0\right)=(0,0)$,

showing that $\left(r_{2}, s_{2}\right) \mathcal{M} \bar{\alpha}\left(r_{1}, s_{1}\right)=0$. Therefore $D$ is a right $\bar{\alpha}$-skew RM ring.

Conversely, suppose that $D$ is central $R M$ and let $a M b=0$ for $a, b \in R$ and for a maximal ideal $M$ of R. Then

$$
a(r+s) b=0
$$

for any $(r, s) \in \mathcal{M}(D)$. This yields

$$
(a, 0)(r, s)(b, 0)=0
$$

for $(r, s) \in \mathcal{M}(D)$. Since $D$ is central RM, therefore

$$
(b, 0)(r, s)(a, 0) \in Z(D)
$$

and so, $b(r+s) a \in Z(D)$, proving that $R$ is central $R M$.

Rege and Chhawchharia called a ring R Armendariz [35, Definition 1.1] if whenever the product of any two polynomials in $R[x]$ over $R$ is zero, then so is the product of any pair of coefficients from two polynomials. It is well-known that any reduced ring is Armendariz, but not conversely. Quasi-Armendariz rings [15] are the generalization of Armendariz rings defined by Hireno. A ring $R$ is called quasi-Armendariz [15] if whenever $f(x)=\sum_{i=0}^{n} a_{i} x^{i}, g(x)=\sum_{j=0}^{m} b_{j} x^{j} \in R[x]$ satisfy $f(x) R[x] g(x)=0$, then $a_{i} R b_{j}=0$ for all $i, j$.

Theorem 2.12. Let $\mathrm{R}$ be a quasi-Armendariz central $R M$ ring. Then $\mathrm{R}[\mathrm{x}]$ is central $R M$ ring.

Proof. Let $p(x) M[x] q(x)=0$ for $\left.p(x)=\sum_{i=0}^{n} a_{i} x^{i}, q(x)=\sum_{j=0}^{m} b_{j} x^{j} \in R[x]\right)$ and for a maximal ideal $M[x]$ of $R[x]$. Since $R$ is a quasi-Armendariz then we obtain $a_{i} M b_{j}=0$ for all $i, j$ and for a maximal ideal $M$ of $R$. Since $R$ is central $R M$, therefore $g(x) M[x] p(x) \subset Z(R[x])$ and hence $R[x]$ is central RM.

The quasi-Armendariz property and central RM property do not imply each other as shown in the next example:

(i) Since $\mathbb{Z}$ is a semiprime ring, therefore by [26, Proposition $2.5(2)(i i)] . T(Z, Z)$ is $R M$. Thus by [26, Example 2.16] the ring $R=\left\{\left(\begin{array}{ll}a & b \\ 0 & a\end{array}\right) \mid a, b \in T(Z, Z)\right\}$. is an $R M$ and hence a central $R M$ ring but not a quasi- Armendariz ring.

(ii) The ring $T_{2}(Z)$ is quasi-Armendariz by [15, Corollary 3.15] but not central RM by Example 2. 


\section{3. $\alpha$-RM Rings}

In this section, the notion of the central $\alpha$-RM ring and it is properties have been introduced. We begin with the following definition.

Definition 3.1. An endomorphism $\alpha$ of a ring $R$ is called $R M$ if whenever $a M b=0$ for $a, b \in R$ and for $a$ maximal ideal $M$ of $R, b M \alpha(a)=0$ (resp., $\alpha(b) M a=0$ ). A ring $R$ is called $\alpha-R M$ if there exists an $R M$ endomorphism $\alpha$ of $R$.

Both simple ring and domains are obviously $\alpha$-RM ring for any endomorphism $\alpha$ of a given ring $R$, but the converses need not be hold. The following example shows that the $\alpha$-RM property is not left-right symmetric.

Consider a ring

$$
R=\left\{\left(\begin{array}{ll}
a & b \\
0 & c
\end{array}\right) \mid a, b, c \in \mathbb{Z}_{2}\right\}
$$

(1) Let $\alpha: R \rightarrow R$ be an endomorphism defined by

$$
\alpha\left(\left(\begin{array}{ll}
a & b \\
0 & c
\end{array}\right)\right)=\left(\begin{array}{ll}
a & 0 \\
0 & 0
\end{array}\right)
$$

For

$$
A=\left(\begin{array}{ll}
a & b \\
0 & c
\end{array}\right) \text { and } B=\left(\begin{array}{cc}
a^{\prime} & b^{\prime} \\
0 & c^{\prime}
\end{array}\right) \in R
$$

We discuss two cases:

Case 1. For a maximal ideal $\mathcal{M}=\left(\begin{array}{ll}0 & R \\ 0 & R\end{array}\right)$ of $R$ assume that $A \mathcal{M} B=0$. Then we have $B M \alpha(A)=0$. It is obvious that $R$ is a right $\alpha-R M$ ring.

Case 2. For a maximal ideal $\mathcal{N}=\left(\begin{array}{ll}R & R \\ 0 & 0\end{array}\right)$ of $R$ by the definition of $\alpha$ assume that $A \mathcal{N} B=0$. Then for any $\left(\begin{array}{ll}r & s \\ 0 & 0\end{array}\right) \in \mathcal{N}$, we have $\operatorname{ara}^{\prime}=0$ and so $\operatorname{ara}^{\prime}=0$. This entails that $B \mathcal{N} \alpha(A)=0$.

Conversely, $\mathrm{R}$ is not left $\alpha$-RM. To see this, let

$$
A=\left(\begin{array}{ll}
0 & 1 \\
0 & 1
\end{array}\right), B=\left(\begin{array}{ll}
1 & 1 \\
0 & 0
\end{array}\right) \in R
$$

Then $A \mathcal{N B}=0$, for the maximal ideal $\mathcal{N}=\left(\begin{array}{ll}R & R \\ 0 & 0\end{array}\right)$ of $R$, but

$$
\left(\begin{array}{ll}
0 & 0 \\
0 & 0
\end{array}\right) \neq\left(\begin{array}{ll}
0 & 1 \\
0 & 0
\end{array}\right)=\left(\begin{array}{ll}
1 & 0 \\
0 & 0
\end{array}\right)\left(\begin{array}{ll}
1 & 0 \\
0 & 0
\end{array}\right)\left(\begin{array}{ll}
0 & 1 \\
0 & 1
\end{array}\right) \in \alpha(B) \mathcal{N A}
$$

(2) Let $\beta: R \rightarrow R$ be an endomorphism defined by

$$
\beta\left(\left(\begin{array}{ll}
a & b \\
0 & c
\end{array}\right)\right)=\left(\begin{array}{ll}
0 & 0 \\
0 & c
\end{array}\right)
$$

By the similar method to the proof (1), we can show that $R$ is left $\beta-R M$, but not right $\beta-R M$.

Lemma 3.2. If a ring $\mathrm{R}$ is an $\alpha$-rigid ring, then $\mathrm{R}$ is $\alpha-R M$. 
Proof. Let $R$ be $\alpha$-rigid. Assume that $a M b=0$ for a maximal ideal $M$ of $R$ and $a, b \in R$. Then $a m b=0$ for all $m \in M$ and so $a b m=0$ since $R$ is reduced and so we obtain $b m \alpha(a) \alpha(b m \alpha(a))=b m \alpha(a b m) \alpha^{2}(a)=$ 0 . This shows that $b m \alpha(a)=0$ and so $b M \alpha(a)=0$ since $R$ is $\alpha$-rigid. Hence $R$ is right $\alpha$-RM. Now by the similar argument to above from $a M b=0$, we also have mab $=0$ for all $m \in M$ since $R$ is reduced. Thus we get $\alpha(\alpha(b) m a) \alpha(b) m a=\alpha^{2}(b) \alpha(m a b) m a=0$. This shows that $\alpha(b) m a=0$ and so $\alpha(b) M a=0$, since $R$ is $\alpha$-rigid. This shows that $R$ is left $\alpha-R M$. Therefore $R$ is $\alpha$-RM.

The converse of Lemma 4.2 does not hold by the following example.

Let $\mathbb{Z}$ be the ring of integers. Consider the ring

$$
R=\left\{\left(\begin{array}{ll}
a & b \\
0 & a
\end{array}\right) \mid a, b \in \mathbb{Z}\right\}
$$

Let $\alpha: R \rightarrow R$ be an endomorphism defined by

$$
\alpha\left(\left(\begin{array}{ll}
a & b \\
0 & a
\end{array}\right)\right)=\left(\begin{array}{cc}
a & -b \\
0 & a
\end{array}\right) .
$$

It is clear that $\alpha$ is an automorphism and $R$ is not semiprime and hence $R$ is not $\alpha$-rigid.

Now for a maximal ideal $\mathcal{M}=\left(\begin{array}{cc}M & R \\ 0 & M\end{array}\right)$ of $R$ and $A, C \in R$, let $A B C=0$ for all $B \in \mathcal{M}$ with

$$
A=\left(\begin{array}{ll}
a & b \\
0 & a
\end{array}\right), C=\left(\begin{array}{ll}
c & d \\
0 & c
\end{array}\right) \text { and } B=\left(\begin{array}{cc}
m & n \\
0 & m
\end{array}\right)
$$

Then we have $a m c=0$ and $a m d+a n c+b m c=0$ for every $m$. This implies that $a=0$ or $c=0$. If $a=0$, then $b m c=0$ for every $m \in M$ a maximal ideal of $\mathbb{Z}$, hence $b=0$ or $c=0$. If $c=0$, then amd $=0$ for each $m \in M$ where $M$ is a maximal ideal of $R$, hence $a=0$ or $d=0$. In both cases, we obtain $C B \alpha(A)=0$. Hence $\mathrm{R}$ is $\bar{\alpha}-\mathrm{RM}$. Therefore $\mathrm{R}$ is $\bar{\alpha}$-RM.

We have the basic equivalence for right $\alpha$-skew RM rings as follows. For a non-empty subset $X$ of a ring $R$, we write $r_{R}(X)=\{c \in R \mid X c=0\}$ which is called the right annihilator of $X$ in $R$. Similarly, $\ell_{R}(X)$ denotes the left annihilator of $X$ in $R$.

Proposition 3.3. For a ring $\mathrm{R}$ with an endomorphism $\alpha$, the following are equivalent:

(1) $\mathrm{R}$ is an $\alpha-R M$ ring.

(2) For a maximal ideal $M$ of $R$ and $a \in R, r_{R}(a M) \subseteq \ell_{R}(M \alpha(a))$.

(3) For a maximal ideal $M$ of $R, A M B=0$ implies $B M \alpha(A)=0$, where $A$ and $B$ are any non-empty subsets of R.

(4) For a maximal ideal $M$ of $\mathrm{R}, \mathrm{IMJ}=0$ implies $\mathrm{JM} \alpha(\mathrm{I})=0$, where $\mathrm{I}$ and $\mathrm{J}$ are any right (or left) ideals of $\mathrm{R}$.

(5) For a maximal ideal $M$ of $\mathrm{R}, \mathrm{IMJ}=0$ implies $\mathrm{JM} \alpha(\mathrm{I})=0$, where $\mathrm{I}$ and $\mathrm{J}$ are any ideals of $\mathrm{R}$.

Proof. $(1) \Leftrightarrow(2)$ and $(3) \Rightarrow(4) \Rightarrow(5)$ are straightforward.

$(2) \Rightarrow(3)$ : Assume that the condition (2) holds. Let $A$ and $B$ be two non-empty subsets of $R$ with $A M B=0$ for a maximal ideal $M$ of $R$. Then $a M b=0$, for any $a \in A$ and $b \in B$ and hence $b M \alpha(a)=0$ by assumption. Thus, $B M \alpha(A)=\sum_{a \in A, b \in B} b M \alpha(a)=0$.

$(5) \Rightarrow(1)$ : Assume that the condition (5) holds. Let $a M b=0$, for a maximal ideal $M$ of $R$ and $a, b \in R$. Then $a R M R b \subseteq a M b=0$ and so, $(\operatorname{RaR}) M(R b R)=0$. By assumption, we have $(R b R) M \alpha(R a R)=0$ and hence $b M \alpha(a)=0$. Thus, $R$ is right $\alpha$ - skew RM.

Proposition 3.4. Let $\alpha$ be an endomorphism of a ring $R$. If $R$ is an $\alpha$-skew $R M$ ring, then $a M b=0$ for a maximal ideal $M$ of $\mathrm{R}$ and $\mathrm{a}, \mathrm{b} \in \mathrm{R}$ implies $\mathrm{aM} \alpha^{2 \mathrm{k}}(\mathrm{b})=0$ and $\alpha^{2 \mathrm{k}-1}(\mathrm{~b}) \mathrm{Ma}=0$, for a positive integer $\mathrm{k}$. 
Proof. Suppose that $R$ is $\alpha$-skew $R M$ and $a M b=0$ for a maximal ideal $M$ of $R$ and $a, b \in R . \alpha(b) M a=$ 0 , which implies $a M \alpha^{2}(b)=0$ and $\alpha^{3}(b) M a=0$ and $a M \alpha^{n}(b)=0, \ldots$, and so we get $a M \alpha^{2 k}(b)=0$ and $\alpha^{2 k-1}(b) M a=0$ for $k \geqslant 1$ inductively.

Proposition 3.5. Let $\alpha$ be an endomorphism of a ring $R, S$ a ring and suppose that $\sigma: R \rightarrow S$ is a ring isomorphism. Then $\mathrm{R}$ is an $\alpha-R M$ ring if and only if $\mathrm{S}$ is an $\sigma \alpha \sigma^{-1}-R M$ ring.

Proof. Let $R$ be an $\alpha-R M$ and let $a^{\prime} N^{\prime} b^{\prime}=0$, for a maximal ideal $N^{\prime}$ of $S$ and $a^{\prime}, b^{\prime} \in S$, then there exist $a, b \in R$ such that $\sigma(a)=a^{\prime}, \sigma(b)=b^{\prime}$ and a maximal ideal $N$ of $R$ such that $\sigma(N)=N^{\prime}$.

So $a^{\prime} N^{\prime} b^{\prime}=0$ implies $\sigma(a) \sigma(N) \sigma(b)=\sigma(0)$ and $\sigma(a N b)=\sigma(0)$ so $a N b=0$ since $\sigma$ is an isomorphism. Then $b N \alpha(a)=0$ by hypothesis. This entails that

$$
0=\sigma(0)=\sigma(b N \alpha(a))=\sigma\left(b N \alpha\left(\left(\sigma^{-1} \sigma\right)(a)\right)\right)=\sigma(b) \sigma(N) \sigma\left(\alpha\left(\sigma^{-1}(\sigma(a))\right)=b^{\prime} N^{\prime}\left(\sigma \alpha \sigma^{-1}\right)\left(a^{\prime}\right)=0 .\right.
$$

Thus $S$ is an $\sigma \alpha \sigma^{-1}-\mathrm{RM}$ ring.

Conversely, let $S$ be an $\sigma \alpha \sigma^{-1}-R M$, let $a M b=0$ for $a, b \in R$ and a maximal ideal $M$ of $R$. Set $\sigma(a)=a^{\prime}, \sigma(b)=b^{\prime}$ and $\sigma(M)=M^{\prime}$. Then $a^{\prime}, b^{\prime} \in S$ and $M^{\prime}$ a maximal ideal of $S$ such that $a^{\prime} M^{\prime} b^{\prime}=$ $\sigma(a M b)=0$. Since $S$ is an $\sigma \alpha \sigma^{-1}-R M$ we have $0=b^{\prime} M^{\prime}\left(\sigma \alpha \sigma^{-1}\right)\left(a^{\prime}\right)=0$ and so $\sigma(b M \alpha(a))=0$. Since $\sigma$ is an isomorphism $b M \alpha(a)=0$. Shows that $R$ is an $\alpha$-RM ring.

For an endomorphism $\alpha$ and an idempotent $e$ of a ring $R$ let $\alpha(e)=e$. Then we have an endomorphism $\bar{\alpha}: e \operatorname{Re} \rightarrow e \operatorname{Re}$ defined by $\bar{\alpha}($ ere $)=e \alpha(r) e$.

Proposition 3.6. Let $\mathrm{R}$ be a ring with an endomorphism $\alpha$ such that $\alpha(e)=e$ for $e^{2}=e \in R$.

(1) If $R$ is an $\alpha-R M$ ring then $e$ Re is an $\bar{\alpha}-R M$ ring for all $e \in \operatorname{Id}(R)$.

(2) If $e$ is central for $e \in I d(R)$, then $R$ is an right $\alpha-R M$ ring if and only if eR and $(1-e) R$ are $\bar{\alpha}-R M$ rings.

Proof. (1) Let $R$ be an $\alpha-R M$ ring and $e \in \operatorname{Id}(R)$. Suppose $a M b=0$ for $a, b \in e$ Re and a maximal ideal $M$ of $e$ Re. Note that $e a e=a$ and $e b e=b$. By claim, there exists a maximal ideal $N$ of $R$ such that $M=e N e$. Hence, $0=a M b=a e N e b=e a e(e N e) e b e=e a e N e b e=a N b=b N \alpha(a)$, sine $R$ is an $\alpha-R M$ ring. Thus $0=b N \alpha(a)=e b e(e N e) e \alpha(a) e=e b e M \bar{\alpha}(e a e)=b M \bar{\alpha}(a)$. Showing that $e$ Re is an $\bar{\alpha}-R M$ ring.

(2) Let $e \in \operatorname{Id}(R)$ be central. Suppose that $e R$ and $(1-e) R$ are $\bar{\alpha}-R M$ rings, let $a M b=0$ for $a, b \in R$ and a maximal ideal $M$ of $R$. Recall that $e M$ and $(1-e) M$ are maximal ideals of $e R$ and $(1-e) R$, respectively. By the proof of $(1)$. Then $e a(e M) e b=0$ and $(1-e) a((1-e) M)(1-e) b=0$. By hypothesis, we have $0=e b(e M) \bar{\alpha}(e a)=e b(e M) e \alpha(a)=e b M \alpha(a)$ and $0=(1-e) b((1-e) M) \bar{\alpha}((1-e) a)=(1-e) b((1-$ $e) M)((1-e) \alpha(a))=(1-e) b M \alpha(a)$.

Thus $b M \alpha(a)=e b M \alpha(a)+(1-e) b M \alpha(a)=0$, showing that $R$ is an $\alpha-R M$ ring.

The converse part follows from (1) directly.

The next Theorem gives the relationship between RM and $\alpha$-RM rings under the condition that $R$ is $\alpha$-compatible.

Theorem 3.7. Let $\mathrm{R}$ be an $\alpha$-compatible ring.

(1) $R$ is reflexive ring if and only if $R$ is $\alpha$-skew reflexive ring.

(2) $R$ is $R M$ ring if and only if $R$ is $\alpha-R M$ ring.

Proof. (1) Let $R$ be a reflexive ring and $a R b=0$ for $a, b \in R$ then $b R a=0$. So $a r b=0$ for all $r \in R$ and bra $=0$ by hypothesis and $b c \alpha(a)=0$ for all $c \in R$ (by $\alpha$-compatibility), hence $b R \alpha(a)=0$, and so $R$ is right $\alpha$-skew reflexive ring. By the same method in above we get $R$ is left $\alpha$-skew reflexive ring. Therefore $\mathrm{R}$ is $\alpha$-skew reflexive ring. 
Conversely, let $R$ be an $\alpha$-skew reflexive ring and $a R b=0$ for $a, b \in R$, so $a c b=0$ for all $c \in R$. Then $b c \alpha(a)=0$ by hypothesis and bca $=0$ for all $c \in R$ (by $\alpha$-compatibility), so $b R a=0$. Therefore $R$ is reflexive ring.

(2) Let $R$ be an $R M$ ring, $a M b=0$ for $a, b \in R$ and a maximal ideal $M$ of $R$, then $a m b=0$ for all $\mathrm{m} \in M$. Then $\mathrm{bma}=0$ for all $\mathrm{m} \in M$ by hypothesis and $b \mathrm{~m} \alpha(a)=0$ for all $m \in M$ (by $\alpha$-compatibility), hence $b M \alpha(a)=0$, and so $R$ is an $\alpha$-RM ring. and hence $R$ is $\alpha$-RM ring.

Conversely, let $R$ be an $\alpha-R M$ ring, $a M b=0$ for $a, b \in R$ and a maximal ideal $M$ of $R$, so $a m b=0$ for all $m \in M$. Then $b m \alpha(a)=0$ by hypothesis and $b m a=0$ for all $m \in M($ by $\alpha$-compatibility), so $b M a=0$. Therefore $\mathrm{R}$ is an $\mathrm{RM}$ ring.

According to the the following Example, we see that the condition " $\alpha$-compatibility "is not superfluous.

(1) Consider the ring $R=\mathbb{Z}_{2} \oplus \mathbb{Z}_{2}$ and it is maximal ideal $\mathcal{M}=\mathbb{Z}_{2} \oplus\{0\}$ with endomorphism $\alpha: R \rightarrow R$ defined by $\alpha((a, b))=(b, a)$ with the usual addition and multiplication $\alpha$ is not compatible. The ring $R$ is a commutative semiprime ring and hence it is an RM ring. However, $R$ is not an $\alpha$-RM ring. Indeed, for $a=(0,1), b=(1,0) \in R$ and $(1,0) \in \mathcal{M}$, we have $(0,1)(1,0)^{2}=0$, but $(1,0)^{3}=(1,0) \neq 0 \in b \mathcal{M} \alpha(a)$.

(2) Consider the ring $R=\mathbb{Z}_{2} \oplus \mathbb{Z}_{2}$ with endomorphism $\alpha: R \rightarrow R$ defined by $\alpha((a, b))=(b, a)$ with the usual addition and multiplication $\alpha$ is not compatible. The ring $R$ is a commutative semiprime ring and hence it is reflexive. However, $R$ is not an $\alpha$-skew reflexive. Indeed, for $a=(0,1), b=(1,0) \in R$ and $(1,1) \in R$, we have $(0,1)(1,1)(1,0)=0$, but $(1,0)(1,1)(1,0)=(1,0) \neq 0 \in b R \alpha(a)$.

Proposition 3.8. Let $\mathrm{R}$ be a ring with endomorphism $\alpha$. Then $\mathrm{R}$ is an $\alpha-R M$ ring if and only if $M a t_{n}(R)$ is an $\bar{\alpha}-R M$ ring for all $\mathrm{n} \geqslant 2$.

Proof. Suppose that $R$ is an $\alpha$-RM ring. Let $S=M_{n}(R)$ and $A N B=0$ for ideals $A, B$ of $S$ and for a maximal idea $N$ of $S$. Using an elementary ring theoretic argument, there exists ideals I and $J$ and a maximal ideal $M$ of $R$ such that $A=M_{n}(I), B=M_{n}(J)$ and $N=M_{n}(M)$. Then

$$
M_{n}(I M J)=M_{n}(I) M_{n}(M)_{n}(J)=A N B=0,
$$

implies IMJ $=0$. Since $R$ is an $\alpha$-RM ring, $J M \alpha(I)=0$ by Proposition 3.3(5). This yields $B N \bar{\alpha}(A)=0$, and so $S$ is an $\bar{\alpha}-R M$ ring for all $n \geqslant 2$ by Proposition 3.3(5).

Conversely, Since $R \cong R e_{11}=e_{11} M_{n}(R) e_{11}$, where $e_{11} \in \operatorname{Id}\left(M_{n}(R)\right)$ so $R$ is an $\alpha$-RM ring by Proposition 3.6(1).

Given a ring $R$ and an $(R, R)$-bimodule $M$, the trivial extension of $R$ by $M$ is the $\operatorname{ring} T(R, M)=R \oplus M$ with the usual addition and the following multiplication: $\left(r_{1}, m_{1}\right)\left(r_{2}, m_{2}\right)=\left(r_{1} r_{2}, r_{1} m_{2}+m_{1} r_{2}\right)$. This is isomorphic to the ring of all matrices $\left(\begin{array}{cc}r & m \\ 0 & r\end{array}\right)$, where $r \in R$ and $m \in M$ and the usual matrix operations are used. by

For an endomorphism $\alpha$ of a ring $R$ and the trivial extension $T(R, R)$ of $R, \bar{\alpha}: T(R, R) \rightarrow T(R, R)$ defined

$$
\bar{\alpha}\left(\left(\begin{array}{ll}
a & b \\
0 & a
\end{array}\right)\right)=\left(\begin{array}{cc}
\alpha(a) & \alpha(b) \\
0 & \alpha(a)
\end{array}\right)
$$

is an endomorphism of $T(R, R)$. Since $T(R, 0)$ is isomorphic to $R$, we can identify the restriction of $\bar{\alpha}$ by $\mathrm{T}(\mathrm{R}, 0)$ to $\alpha$.

The converse of Proposition 3.6 does not hold in general. 
Corollary 3.9. Let $\mathrm{R}$ be a reduced ring with an endomorphism $\alpha$. Then the following are equivalent.

(1) $R$ is an $\alpha-R M$ ring.

(2) The trivial extension $T(R, R)$ of $R$ is an $\bar{\alpha}-R M$ ring.

(3) $\frac{\mathrm{R}[\mathrm{x}]}{\mathrm{x}^{\mathrm{n}} \mathrm{R}[\mathrm{x}]}$ is an $\bar{\alpha}-R M$ ring, for $\mathrm{n} \geqslant 2$.

Proof. (1) $\Rightarrow$ (3). It follows by the [2, Theorem 3.1]. (3) $\Rightarrow$ (2). The proof is trivial. (2) $\Rightarrow$ (1). Suppose that $T(R, R)$ of any non-zero ring $R$ is an $\bar{\alpha}-R M$ ring and let $a M b=0$ for $a, b \in R$ and a maximal ideal $M$ of R. Then,

$$
\left(\begin{array}{cc}
M & R \\
0 & M
\end{array}\right)
$$

is a maximal ideal of $T(R, R)$ and

$$
\left(\begin{array}{ll}
a & 0 \\
0 & a
\end{array}\right)\left(\begin{array}{cc}
M & R \\
0 & M
\end{array}\right)\left(\begin{array}{ll}
0 & b \\
0 & 0
\end{array}\right)=\left(\begin{array}{cc}
0 & a M b \\
0 & 0
\end{array}\right)=\left(\begin{array}{ll}
0 & 0 \\
0 & 0
\end{array}\right) .
$$

Since $T(R, R)$ is an $\bar{\alpha}-R M$ then,

$$
\left(\begin{array}{ll}
0 & b \\
0 & 0
\end{array}\right)\left(\begin{array}{cc}
M & R \\
0 & M
\end{array}\right)\left(\begin{array}{cc}
\alpha(a) & 0 \\
0 & \alpha(a)
\end{array}\right)=\left(\begin{array}{cc}
0 & b M \alpha(a) \\
0 & 0
\end{array}\right)=\left(\begin{array}{ll}
0 & 0 \\
0 & 0
\end{array}\right),
$$

and so $b M \alpha(a)=0$. Thus, $R$ is an $\alpha-R M$ ring.

Proposition 3.10. If $\mathrm{R}$ is a division ring, then $\mathrm{T}(\mathrm{R}, \mathrm{R})$ is an $\bar{\alpha}-R M$.

Proof. Let $\mathrm{R}$ be a division ring. Then

$$
M=\left(\begin{array}{ll}
0 & R \\
0 & 0
\end{array}\right)
$$

is the only maximal ideal of $T(R, R)$. Suppose that $A M B=0$, for

$$
A=\left(\begin{array}{ll}
a & b \\
0 & a
\end{array}\right), B=\left(\begin{array}{ll}
c & d \\
0 & c
\end{array}\right) \in T(R, R) .
$$

Then, we have

$$
\left(\begin{array}{ll}
a & b \\
0 & a
\end{array}\right)\left(\begin{array}{ll}
0 & R \\
0 & 0
\end{array}\right)\left(\begin{array}{ll}
c & d \\
0 & c
\end{array}\right)=\left(\begin{array}{ll}
0 & 0 \\
0 & 0
\end{array}\right)
$$

implies arc $=0$. Since $R$ is division ring so $R$ is domain and $\operatorname{arc}=0$ implies $a=0$ or $r c=0$ so we have $a=0$ or $r=0$ or $c=0$ and so from

$$
\left(\begin{array}{ll}
c & d \\
0 & c
\end{array}\right)\left(\begin{array}{ll}
0 & R \\
0 & 0
\end{array}\right)\left(\begin{array}{cc}
\alpha(a) & \alpha(b) \\
0 & \alpha(a)
\end{array}\right)=\left(\begin{array}{ll}
0 & 0 \\
0 & 0
\end{array}\right)
$$

we get $\operatorname{cr} \alpha(a)=0$ and this implies that $B \mathcal{M} \bar{\alpha}(A)=0$. Thus, $T(R, R)$ is an $\bar{\alpha}-R M$ ring.

Let $R$ be an algebra over a commutative ring $S$. Due to Dorroh [13], the Dorroh extension of $R$ by $S$ is the Abelian group $R \oplus S$ with multiplication given by $\left(r_{1}, s_{1}\right)\left(r_{2}, s_{2}\right)=\left(r_{1} r_{2}+s_{1} r_{2}+s_{2} r_{1}, s_{1} s_{2}\right)$ for $r_{i} \in R$ and $s_{i} \in S$. We use $D$ to denote the Dorroh extension of $R$ by $S$.

For an endomorphism $\alpha$ of $R$ and the Dorroh extension $D$ of $R$ by $S, \bar{\alpha}: D \rightarrow$ D defined by $\bar{\alpha}(r, s)=$ $(\alpha(r), s)$ is an S-algebra homomorphism. Recall that an algebra $R$ in which every element of the algebra is nilpotent a nil algebra.

The next result shows one way to build new $\alpha$-RM rings from old once. 
Theorem 3.11. Let $\mathrm{R}$ be a nil algebra over a field $\mathrm{K}$ and $\mathrm{D}$ the Dorroh extension of $\mathrm{R}$ by $\mathrm{K}$. Let $\alpha$ be an endomorphism of $\mathrm{R}$ with $\alpha(1)=1$. Then $\mathrm{R}$ is a right $\alpha$-skew reflexive ring if and only if $\mathrm{D}$ is an $\bar{\alpha}-R M$ ring.

Proof. First, note that $\mathcal{M}=R \oplus\{0\}$ is the unique maximal ideal of $D$, since every $(r, s) \in D$ is a unit when $s \neq 0$ by the proof of [19, Proposition 1.5] and that $s \in K$ is identified with $s 1 \in R$ and so $R=\{r+s \mid$ $(\mathrm{r}, \mathrm{s}) \in \mathrm{D}\}$.

Suppose that $R$ is a right $\alpha$-skew reflexive ring with $\alpha(1)=1$ and let $\left(r_{1}, s_{1}\right) \mathcal{M}\left(r_{2}, s_{2}\right)=0$ for $\left(r_{1}, s_{1}\right),\left(r_{2}, s_{2}\right) \in D$. Since

$$
\left(r_{1}, s_{1}\right)(r, 0)\left(r_{2}, s_{2}\right)=\left(r_{1} r r_{2}+s_{1} r r_{2}+s_{2} r_{1} r+s_{1} s_{2} r, 0\right)=(0,0),
$$

for $r_{1}, r, r_{2} \in R, s_{1}, s_{2} \in K$ and

$$
\left(r_{1}+s_{1} 1\right) r\left(r_{2}+s_{2} 1\right)=r_{1} r r_{2}+s_{1} r r_{2}+s_{2} r_{1} r+s_{1} s_{2} r
$$

for $\left(r_{1}+s_{1} 1\right),\left(r_{2}+s_{2} 1\right) \in R$ and $r \in R$.

For any $(r, 0) \in \mathcal{M}$, we have $\left(r_{1}, s_{1}\right) R\left(r_{2}, s_{2}\right)=0$. Since $R$ is a right $\alpha$-skew reflexive ring, we get

$$
0=\left(r_{2}+s_{2} 1\right) R \alpha\left(r_{1}+s_{1} 1\right)=\left(r_{2}+s_{2} 1\right) R\left(\alpha\left(r_{1}\right)+s_{1}\right) .
$$

Hence

$$
r_{2} r \alpha\left(r_{1}\right)+s_{2} r \alpha\left(r_{1}\right)+s_{1} r_{2} r+s_{1} s_{2} r=0,
$$

for all $r \in R$. Let $(r, 0) \in \mathcal{M}$. Then

$\left(r_{2}, s_{2}\right)(r, 0)\left(\alpha\left(r_{1}\right), s_{1}\right)=\left(\left(r_{2} r+s_{2} r\right) \alpha\left(r_{1}\right)+s_{1}\left(r_{2} r+s_{2} r\right), 0\right)=\left(r_{2} r \alpha\left(r_{1}\right)+s_{2} r \alpha\left(r_{1}\right)+s_{1} r_{2} r+s_{1} s_{2} r, 0\right)=(0,0)$,

showing that $\left(r_{2}, s_{2}\right) \mathcal{M} \bar{\alpha}\left(r_{1}, s_{1}\right)=0$. Therefore $D$ is a right $\bar{\alpha}-R M$ ring.

Conversely, suppose that $D$ is a right $\bar{\alpha}-R M$ and let $a R b=0$ for $a, b \in R$. Then

$$
(a, 0) \mathcal{M}(b, 0)=(a R b, 0)=(0,0) .
$$

Since $\mathrm{D}$ is a right $\bar{\alpha}-\mathrm{RM}$ ring,

$$
(b, 0) \mathcal{M} \bar{\alpha}(a, 0)=(b, 0) \mathcal{M}(\oslash(\dashv), \prime)=\prime,
$$

and it implies that $b R \alpha(a)=0$. Thus $R$ is a right $\alpha$-skew reflexive.

\section{Central $\alpha$-RM Rings}

In this section, the notion of the central $\alpha$-RM ring and it is properties have been introduced. We begin with the following definition

Definition 4.1. An endomorphism $\alpha$ of a ring $R$ is called central $R M$ if whenever $a M b=0$ for $a, b \in R$ and for a maximal ideal $M$ of $R, b M \alpha(a) \subset Z(R)$. A ring $R$ is called central $\alpha-R M$ if there exists a central $\mathrm{RM}$ endomorphism $\alpha$ of R. 
From definition it is clear that every central $R M$ ring $R$ is central $i d_{R}-R M$ ring. Every $\alpha-R M$ ring is central $\alpha-R M$ ring. But the converse is not true by the following example.

Consider the ring $R$ as in Example 1. Clearly $R$ is central $i d_{R}-R M$ ring but not $i d_{R}-R M$ ring.

Recall that if $\alpha$ is an endomorphism of a ring $R$, then the map $\bar{\alpha}: R[x] \Rightarrow R[x]$ defined by $\bar{\alpha}\left(\sum_{i=0}^{m} a_{i} x^{i}\right)=$ $\sum_{i=0}^{m} \alpha\left(a_{i}\right) x^{i}$ is an endomorphism of the polynomial ring $R[x]$ and clearly this map extend $\alpha$. Let $R$ be a ring and $\Delta$ be a multiplicative monoid in $\mathrm{R}$ consisting of central regular elements, and let $\Delta^{-1} \mathrm{R}=$ $u^{-1} a \mid u \in \Delta, a \in R$, then $\Delta^{-1} R$ is a ring. We have

Proposition 4.2. Let $\Delta$ be a multiplicatively closed subset of a ring $\mathrm{R}$ consisting of central regular elements and $\mathrm{F}(\mathrm{x}) \mathrm{H}(\mathrm{x}) \mathrm{G}(\mathrm{x})=0$. Then $\mathrm{R}[\mathrm{x}]$ is central $\alpha-R M$ if and only if $\Delta^{-1} \mathrm{R}[\mathrm{x}]$ is central $\bar{\alpha}-R M$.

Proof. It sufficed to show that $\Delta^{-1} R[x]$ is central $\bar{\alpha}-R M$ if $R[x]$ is central $\alpha$-RM. Let $f(x)=\sum_{i=0}^{m} u_{i}^{-1} a_{i} x^{i}$, $g(x)=\sum_{j=0}^{n} v_{j}^{-1} b_{j} x^{j} \in \Delta^{-1} R[x]$ and for $h(x) \in \Delta^{-1}(\mathcal{M} R)[x]$ where $\Delta^{-1}(\mathcal{M} R)[x]$ is a maximal ideal of $\Delta^{-1} R[x]$. Then $\left.F(x)=\left(u_{m}, u_{m-1}, \cdots, u_{0}\right) f(x), H(x)=\left(\lambda_{t}, \lambda_{t-1}, \cdots, \lambda_{0}\right) h(x)\right)$ and $G(x)=\left(v_{n}, v_{n-1}, \cdots, v_{0}\right) g(x) \in$ $R[x]$. Since $R[x]$ is central $\alpha-R M$ and $F(x) H(x) G(x)=0$, this implies that $G(x) H(x) \alpha(F(x)) \subset Z(F(x) H(x) G(x))$, and so $g(x) h(x) \alpha(f(x)) \subset Z(R[x])$ since $\Delta$ is a multiplicative monoid in $R$ consisting of central regular elements and $u_{i}, v_{j} \in \Delta$ for all $i, j$. This shows that $\Delta^{-1} R[x]$ is central $\bar{\alpha}$-RM.

Proposition 4.3. For a ring $R$. Then $R[x]$ is central $\alpha-R M$ if and only if $R\left[x, x^{-1}\right]$ is central $\alpha-R M$.

Proof. It suffices to prove the necessity only, Suppose $R[x]$ is central $\alpha$-RM. Let $\Delta=1, x, x^{2}, \ldots$, Then clearly $\Delta$ is a multiplicative closed subset of $R[x]$ and $R\left[x, x^{-1}\right]=\Delta^{-1} R[x]$. It follows that $R\left[x, x^{-1}\right]$ is central $\alpha$-RM, by Proposition 4.2.

Theorem 4.4. Let $\mathrm{R}$ be a quasi-Armendariz ring. Then the following statements are equivalent:

(1) $R$ is central $\alpha-R M$.

(2) $R[x]$ is a central $\bar{\alpha}-R M$.

(3) $R\left[x, x^{-1}\right]$ is a central $\bar{\alpha}-R M$.

Proof. It is enough to show that $(i) \Rightarrow(i i)$ : Let $f(x)=\sum_{i=0}^{m} a_{i} x^{i}, g(x)=\sum_{j=0}^{n} b_{j} x^{j} \in R\left[\left[x, x^{-1}\right]\right.$, and $h(x) \in$ $(M R)[x]$ such that Let $f(x) h(x) g(x)=0$. Since $R$ is quasi-Armendariz, then $a_{i} R b_{j}=0$ for all $i, j$ and so $b_{j} R \alpha\left(a_{i}\right) \in Z(R)$. Therefore $g(x)(M R)[x] \alpha(f(x)) \in Z(R[x])$. Hence, $R[x]$ is central $\alpha$-skew $R M$.

If you'd like to thank anyone, place your comments here.

\section{References}

[1] A.M. Abdul-Jabbar, C. A. K. Ahmad, T.K.Kwak and Y. Lee,Reflexivity with maximal ideal axes, Comm. Algebra 45, (2017), 4348-4361. https:/ / doi.org/10.1080/00927872.2016.1222398

[2] A. M. Abdul-Jabbar, C. A. K. Ahmad, Skew reflexive property with maximal ideal axes The Zanco Journal of Pure and Applied in theSalahaddin University. Vol 29 No 3 (2017) . https://doi.org/10.21271/zjpas.29.3.9

[3] N.Agayev, A. harmannci and S.Halicioglu, Extended Armendariz rings, Algebra Group Geom. 26(4) (2009) 343-354.

[4] E. P. Armendariz, A note on extensions of Baer and P.P.-rings, J.Austral. Math. Sco. 18(1974), 470-473.

[5] D.D. Anderson and V.Camillo, semi groups and rings whose zero products commute, Comm. Algebra 27(6)(1999), 2847-2852.

[6] M.Başer, C. Y. Hong and T. K. . Kwak, ON Extended Reversible Rings, Algebra Colloq. 16 (2009), 37-48. https://doi.org/10.1142/s1005386709000054

[7] M.Başer and T. K. . Kwak, Extended semicommutative Rings, Algebra Colloq. 17 (2010), $257-264$. https:/ / doi.org/10.1142/s1005386710000271 
[8] H. E. Bell, Near-rings in which each element is a power of itself, Bull. Austral. Math. Soc. 2 (1970), 363-368. https: / / doi.org/10.1017/s0004972700042052

[9] G.F.Bikenmeimer, J.Y.Kim and J.K.Park, Principally quasi-Baer rings, Comm. Algebra 29(2001), 639-660.

[10] G.F.Bikenmeimer, J.Y.Kim and J.K.Park, Polynomial extensions of Baer and quasi-Baer rings, J.Pure. Apll. Algebra 159(2001), 25-42. https: / / doi.org/10.1016/s0022-4049(00)00055-4

[11] V. Camillo, T. K. Kwak and Y. Lee, Ideal-symmetric and semiprime rings, Comm. Algebra 41(2013), $4504-4519$. https:/ / doi.org/10.1080/00927872.2012.705402

[12] P.M. Cohn, Reversible rings, Bull. London Math. Soc. 31(1999), 641-648.

[13] J. L. Dorroh, Concerning adjunctins to algebras, Bull. Amer. Math. Soc. 38 (1932), 85-88.

[14] E. Hashemi, A. Moussavi, Polynomial extensions of quasi-Baer rings, Acta. Math. Hungar, 151(2000),215-226.

[15] Y. Hirano, On annihilator ideals of a polynomial ring over a non commutative ring, J. Pure Appl. Algebra 168 (2002), 215-226. https: / / doi.org/10.1016/s0022-4049(01)00053-6

[16] C. Y. Hong, N. K. Kim and T. K. Kwak, Ore extensions of Baer and p.p.-rings, J. Pure Appl. Algebra 151 (2002), $37-52$. https:/ / doi.org/10.1016/s0022-4049(99)00020-1

[17] C. Huh, Y. Lee and A.Smoktunowice, Armendariz rings and semicommutative rings, Comm.Algebra 30 (2002), 751761. https://doi.org/10.1081/agb-120013179

[18] S. U. Hwang, Y. C. Jeon and Y. Lee, Structure and topological conditions of NI rings, J. Algebra 302 (2006), 186-199. https://doi.org/10.1016/j.jalgebra.2006.02.032

[19] H. L. Jin, D.W. Jung, Y. Lee, S. J. Ryu, H. J. Sung and S. J. Yun, Insertion-of-Factors-Property with factors maximal ideals, J. Korean Math. Soc. (to appear).

[20] D.A. Jordan, Bijective extensions of injective ring endomorphisms, J. Lond. Math. Soc. 25 (1982), 435-448. https:/ / doi.org/10.1112/jlms/s2-25.3.435

[21] G. Kafkas, B. Ungor, H. Halicioglu and A.Harmanci, Generalized symmetric rings, Algebra and Discrete Math. 12 (2011), 72-84.

[22] G. Mason, Reflexive ideals, Comm. Algebra 9 (1981), 1709-1724. https:/ / doi.org/10.1080/00927878108822678

[23] N. K. Kim, Y. Lee,Armendariz rings and reduced rings, J. Algebra223(2000), $477-488$. https:/ / doi.org/10.1006/jabr.1999.8017

[24] J. Krempa, Some example of reduced rings, Algebra Colloq. 3 (1996),289-300.

[25] T. K. Kwak, Extensions of extended symmetric rings, Bull. Korean Math. Soc. 44 (2007), 777-788. https: / / doi.org/10.4134/bkms.2007.44.4.777

[26] T.K. Kwak, Y. Lee, Reflexive property of rings, Comm. Algebra, 40(2012), $1576-1594$. https:/ / doi.org/10.1080/00927872.2011.554474 1, 2

[27] T. K. Kwak, Y. Lee, Reflexive property on idempotents ,Bull. Korean Math. Soc. 50 (2013), 1957-1972. https:/ / doi.org/10.4134/bkms.2013.50.6.1957

[28] T. K. Kwak, Y. Lee and S. J. Yun, Reflexive property skewed by ring endomorphisms, Korean J. Math. 22 (2014), $217-234$. https:/ / doi.org/10.11568/kjm.2014.22.2.217

[29] J.Y.Kim, Certain rings whose simple singular modules are GP-injective, proc. Japan. Acad. Ser 81 (2005), 125-128. https:/ / doi.org/10.3792/pjaa.81.125

[30] J.Y.Kim and Y. Lee, Extensions of reversible rings, J. Pure . Appl. Algebra 185 (2003), $207-223$. https: / / doi.org/10.4153/cmb-1971-065-1

[31] J. Lambek, On the representation of modules by sheaves of factor modules, Canad. Math. Bull. 14(1971) 359-368.

[32] G. Mason, Reflexive ideals, Comm. Algebra 9 (1981), 1709-0724. https:/ / doi.org/10.1080/00927878108822678

[33] J. C. McConnell, J. C. Robson, Non commutative Noetherian Rings, John Wiley \& Son Ltd., 1987.

[34] T. Ozen, N. Agayev and A. Harmanci, On a class of semicommutative rings, Kyungpook Math. J. 51 (2011), $283-291$. https: / / doi.org/10.5666/kmj.2011.51.3.283

[35] M. B. Rege and S. Chhawchharia, Armendariz rings, Proc. Japan Acad. Ser. A. Math. Sci 73 (1997), 14-17.

[36] G. Shin, Prime ideals and sheaf represntations of a pseudo symmetric ring, Trans. Amer. Math. Soc. 184 (1973), $43-69$. https: / / doi.org/10.1090/s0002-9947-1973-0338058-9

[37] L. Zhao. X. Zhu, Extensions of $\alpha$-reflexive rings Asian-Europ. J. Math. 5 (2012) 1250013 (10 pages). https://doi.org/10.1142/s1793557112500131 3 1 